\title{
Kinematics and metallicity relations for dwarf galaxies in the Local Group
}

\author{
Giuseppina Battaglia*, Eline Tolstoy, Amina Helmi \\ Kapteyn Astron. Inst., University of Groningen, The Netherlands \\ E-mail: gbattagleastro.rug.nl, etolstoyeastro.rug.nl \\ ahelmieastro.rug.nl

\section{Mike Irwin} \\ Institute of Astronomy, Cambridge University, $U K$ \\ mikedast.cam.ac.uk
}

\begin{abstract}
We present the first results of intermediate resolution spectral observations in the CaII triplet region obtained with VLT/FLAMES for 3 dwarf spheroidal galaxies in the Local Group: Sculptor, Fornax and Sextans. For each galaxy, we obtained accurate velocity and metallicity measurements for hundreds of stars out to and beyond the tidal radius: this was not possible with previous observations. Therefore, with this work we are able to investigate the presence of gradients in the kinematic and metallicity properties and explore possible links between them.

In each galaxy, we find clear evidence for the presence of two distinct stellar components with different spatial distribution and kinematics: the metal rich component is more centrally concentrated and kinematically colder than the metal poor one. These findings show that even dSphs, the simplest galaxies known, have undergone a complex evolution.
\end{abstract}

Baryons in Dark Matter Halos

5-9 October 2004

Novigrad, Croatia

${ }^{*}$ Speaker. 


\section{Introduction}

Dwarf Spheroidal galaxies (dSphs) are the smallest and faintest galaxies known. Because of their small size, they have been considered as single-cell star-forming systems and the simplest to understand in terms of galaxy evolution (for a review, see Mateo 1998). DSphs are predominantly dominated by stellar populations with age $>10 \mathrm{Gyr}$ (e.g. Sculptor and Sextans), but some of them (e.g. Fornax) have undergone more recent episodes of star formation (2-8 Gyr ago). There has been evidence that there are stellar population gradients caused by two distinct populations or a variation in properties in several dSphs. In a Colour-Magnitude Diagram (CMD) the old population is detectable in the horizonthal branch: the Blue Horizonthal Branch (BHB) contains the oldest stars or the more metal poor and the Red Horizonthal Branch (RHB) the slightly younger or the more metal rich. By analysing the spatial distribution of HB stars it has been found that RHB stars are in general more concentrated than BHB stars in dSph galaxies (Harbeck et al. 2001), which suggests that either age or metallicity gradients (or both) are consistently present in these systems. Only spectroscopic observations can unambiguously separate metallicity gradients and make a link with the kinematics.

Now I am going to tell about my work which will clarify this issue by taking large numbers of spectra of individual stars. As part of the DART large program at ESO, we obtained intermediate resolution FLAMES CaII triplet spectra for Sculptor (Scl), Fornax (Fnx) and Sextans (Sxt) dSphs. For each of these galaxies, we observed hundreds of red giant branch stars out to and beyond the tidal radius (example, Fig.11). Among the observed spectra, we selected only the ones with a signal-to-noise $\mathrm{S} / \mathrm{N}>10$, obtaining accurate velocities $\left(\sim \pm 2 \mathrm{~km} \mathrm{~s}^{-1}\right)$ and $[\mathrm{Fe} / \mathrm{H}](\sim \pm 0.1 \mathrm{dex})$ measurements for about 320, 600 and 230 radial velocity members for Scl, Fnx and Sxt respectively (we consider members the stars with heliocentric velocity between $3 \sigma$ from the systemic velocity).

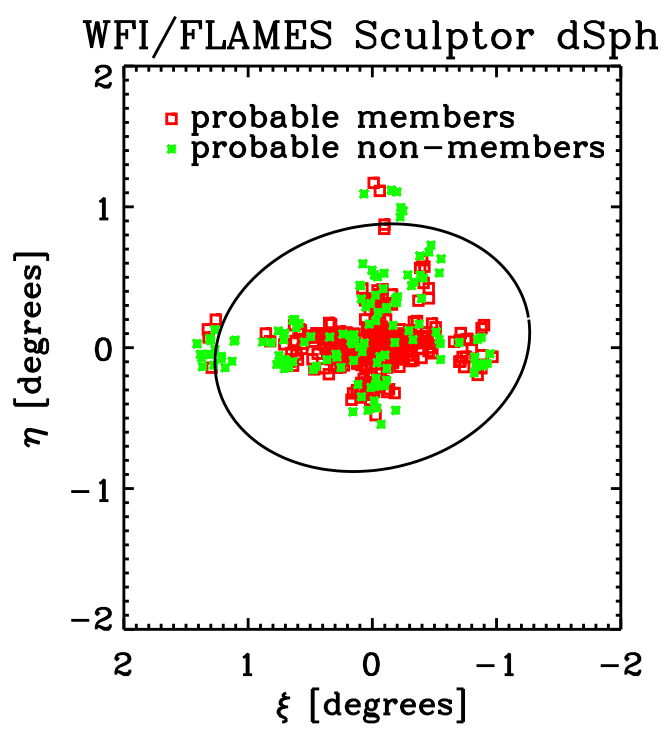

Figure 1: Locations of the observed FLAMES targets for Sculptor (squares: members; small asterisks: nonmembers). The ellipse indicates the tidal radius. Note the presence of probable members beyond the tidal radius 


\section{Metallicity and Kinematics of large stellar samples in dSphs}

Figure 2 shows an histogram of the $[\mathrm{Fe} / \mathrm{H}]$ measurements for the three galaxies at distances lower than 2 core radii (solid) and larger than two $r_{c}$ (dashed). A metallicity gradient is clearly present in all the three galaxies: the average $[\mathrm{Fe} / \mathrm{H}]$ is higher in the central regions and decreases in the outer regions. In Figure 3 we show the velocity distribution at different spatial bins for Scl and Fnx dSphs where the $[\mathrm{Fe} / \mathrm{H}]$ distribution is divided between "metal-poor" and "metal-rich". The metallicity cut $([\mathrm{Fe} / \mathrm{H}]=-1.7$ for $\mathrm{Scl},[\mathrm{Fe} / \mathrm{H}]=-1.4$ for $\mathrm{Fnx})$ corresponds to the peak of the $[\mathrm{Fe} / \mathrm{H}]$ distribution. The two metallicity components have different spatial and kinematic distributions. We can again see that the inner regions are dominated by the metal rich component; at larger radii metal rich stars disappear almost completely. The metal poor population typically exhibits a larger velocity dispersion than the metal rich one. For Scl the division between the dominance of the two different populations appears to occur where there is the change in dominance between RHB and BHB (Tolstoy et al. 2004).

\section{Conclusions}

Our sample consists of three dSphs (Scl, Fnx and Sxt) with different star formation histories, masses and average metallicity. Scl and Sxt are dominated by old stellar populations whilst the bulk of stars in Fnx is represented by intermediate-age stars; the average metallicity (derived in this work) is $[\mathrm{Fe} / \mathrm{H}]=-1.8,-1.4,-2.1$ for $\mathrm{Scl}, \mathrm{Fnx}$ and Sxt respectively. Our analysis of spectra for hundreds of members in each of the three dSphs shows that the metallicity properties of these objects change with radius. We distinguish two components: a "metal-poor" and a "metal-rich" one with different spatial distribution and kinematics. The metal-rich component is more centrally concentrated, less spatially extended than the metal-poor one and, in average, shows a lower velocity dispersion.

The fact that we are observing the same features in galaxies so different from each other might indicate that these are common charactestics among dSphs. Our results suggest that dSphs contain multiple stellar components with different spatial, kinematic and metallicity distributions, and rule out formation scenarios involving single bursts of star formation.

\section{References}

[1] D. Harbeck et al. 2001, AJ, 122, 3092 (astro-ph/0109121)

[2] M. Mateo 1998, ARA\&A, 36, 435 (astro-ph/9810070)

[3] E. Tolstoy et al. 2004, astro-ph/0411029 

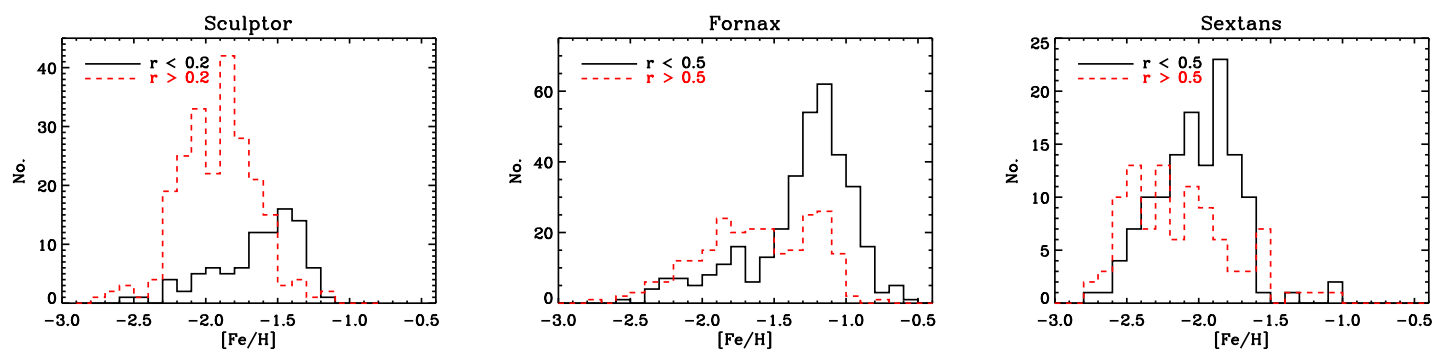

Figure 2: Histogram of the $[\mathrm{Fe} / \mathrm{H}]$ measurements for Scl, Fnx and Sxt for elliptical radii $r<2 r_{\mathrm{c}}$ (solid) and $>2 r_{\mathrm{c}}$ (dashed). The elliptical radius is in degrees.
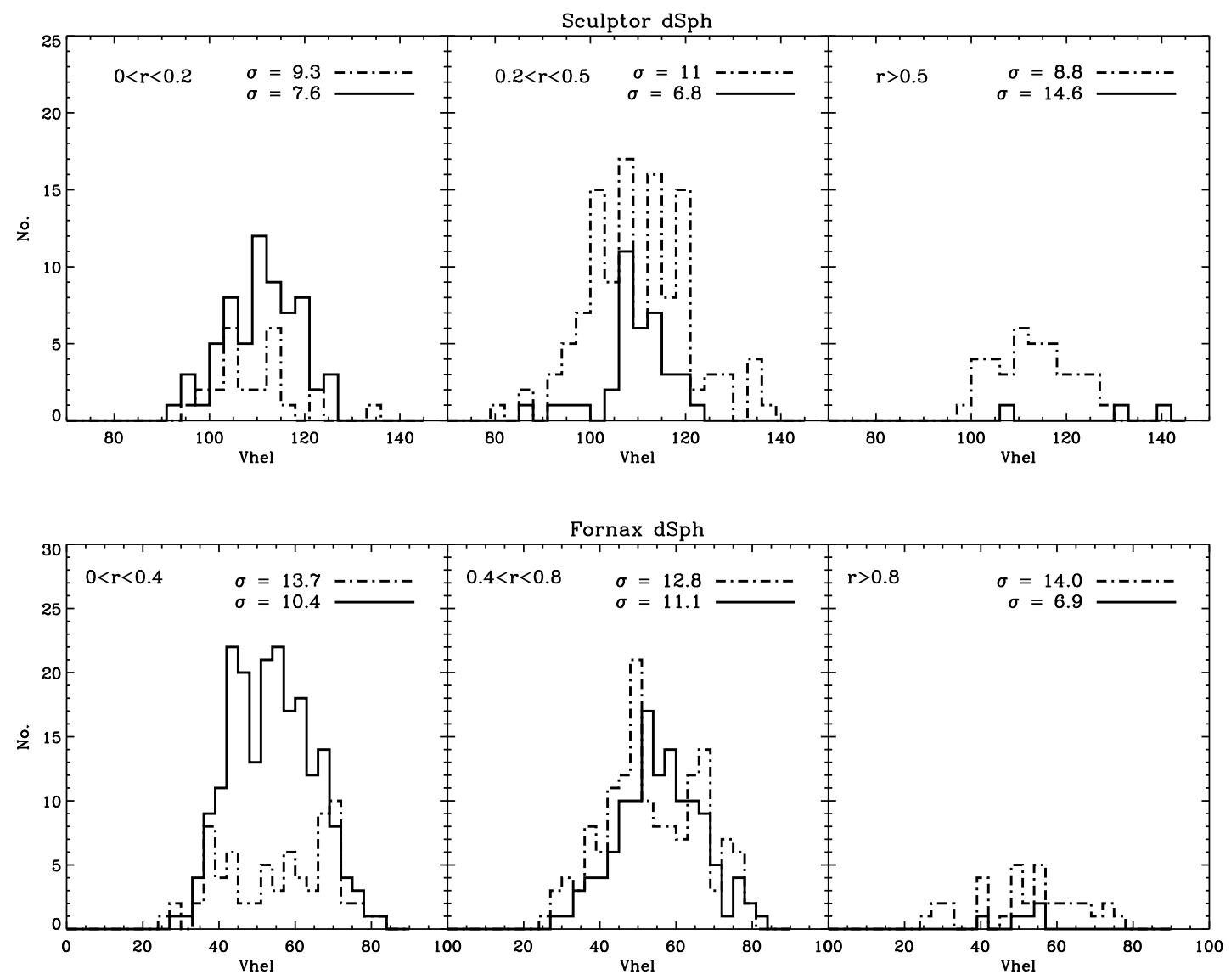

Figure 3: Velocity histogram for different spatial samples for Scl (top) and Fnx (bottom) dSphs. The solid line shows the higher metallicity component and the dot-dashed line the lower metallicity one; $r$ is the elliptical radius in degrees. For $\mathrm{Scl}$ the metallicity cut is at $[\mathrm{Fe} / \mathrm{H}]=-1.7$; for $\mathrm{Fnx}$ at $[\mathrm{Fe} / \mathrm{H}]=-1.4$ 